\title{
PHOTOMETRIC LUNAR SURFACE RECONSTRUCTION
}

\author{
Ara V. Nefian, Oleg Alexandrov, Zachary Moratto, Taemin Kim and Ross A. Beyer \\ NASA Ames Research Center \\ \{ara.nefian, oleg.alexandrov, zachary.moratto, taemin.kim, ross.a.beyer\}@ nasa.gov
}

\begin{abstract}
Accurate photometric reconstruction of the Lunar surface is important in the context of upcoming NASA robotic missions to the Moon and in giving a more accurate understanding of the Lunar soil composition. This paper describes a novel approach for joint estimation of Lunar albedo, camera exposure time, and photometric parameters that utilizes an accurate Lunar-Lambertian reflectance model and previously derived Lunar topography of the area visualized during the Apollo missions. The method introduced here is used in creating the largest Lunar albedo map (16\% of the Lunar surface) at the resolution of 10 meters/pixel.
\end{abstract}

\section{INTRODUCTION}

High resolution and accurate albedo maps of planetary surfaces in general, and the Lunar surface in particular, are used in landing site selection, mission planing, understanding soil composition, and as educational resources. Accurate albedo maps effectively support landing operations as they are used to estimate the landing site appearance under the illumination conditions (Sun and spacecraft position) at landing time. In planetary science the albedo maps advance our understanding of soil porosity, surface composition and formation through correlations with terrain models and other data mapping (multi-spectral, mineralogical) products.

This paper describes a method for albedo map reconstruction using imagery retrieved by the Apollo 15, 16 and 17 missions. The Apollo Metric Camera was flown on an orbit at approximately 100 $\mathrm{km}$ above the Lunar surface. It was a wide field of view $\left(75^{\circ}\right)$ orbital mapping camera that photographed consecutive images with approximately $80 \%$ overlap. The digital scans of these film images $[9,3]$ capture the full dynamic range and resolution of the original film, resulting in digital images of size $22,000 \times 22,000$ pixels, representing a resolution of 10 meters/pixel and covering approximately $16 \%$ of the Lunar surface. Each pixel of the Apollo Metric Camera images was formed by a combination of many factors, including albedo, terrain slope, exposure time, shadowing, and viewing and illumination angles. The goal of albedo reconstruction is to separate the contributions of these factors. This is possible in part because of redundancy in the data; specifically, the same surface location is often observed in multiple overlapping images. The albedo reconstruction technique includes all of the above quantities in an image formation model and re-estimates them from the existing imagery. The overall system is shown in Figure 1. The components of this block diagram are explained in more detail in the following sections.

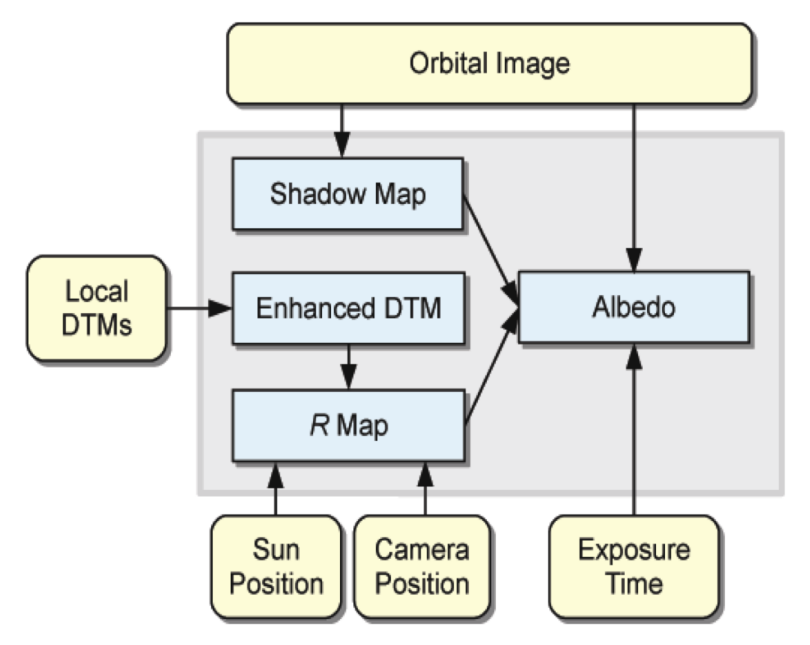

Fig. 1. The overall albedo reconstruction system.

\section{PHOTOMETRIC MODEL}

Starting with the first images from the Apollo missions, a large number of Lunar reflectance models were studied $[6,7,8]$. In this work the reflectance is derived from the Lunar-Lambertian model $[6,5]$. As shown in Figure 2, we define the following unit vectors: $\mathbf{n}$ is the local surface normal, and $\mathbf{l}$ and $\mathbf{v}$ are directed at the locations of the Sun and the spacecraft, respectively, at the time when the image was captured. We further define the angles $\mathbf{i}$ separating $\mathbf{n}$ from $\mathbf{l}$, e separating $\mathbf{n}$ from $\mathbf{v}$, and the phase angle $\alpha$ separating $\mathbf{l}$ from $\mathbf{v}$.

The reflectance model used in our approach is given by

$$
\begin{aligned}
R_{i j}^{k}=\left(e^{-c_{1} \alpha}+c_{2}\right) & {\left[(1-L(\alpha)) \cos \left(\mathbf{i}_{i j}^{k}\right)\right.} \\
& \left.+2 L(\alpha) \frac{\cos \left(\mathbf{i}_{i j}^{k}\right)}{\cos \left(\mathbf{i}_{i j}^{k}\right)+\cos \left(\mathbf{e}_{i j}^{k}\right)}\right]
\end{aligned}
$$

where $L(\alpha)$ is a weighting factor between the Lunar and Lambertian reflectance models [2] that depends on the phase angle and surface properties. $R$ is a photometric function that depends on the angles $\alpha, \mathbf{i}$ and $\mathbf{e}$. The parameters $c_{1}$ and $c_{2}$ play a significant role in modeling the surface reflectance at low phase angles. This can be noticed in the reduction of the banding artifacts in Figure 8 on the top right. 


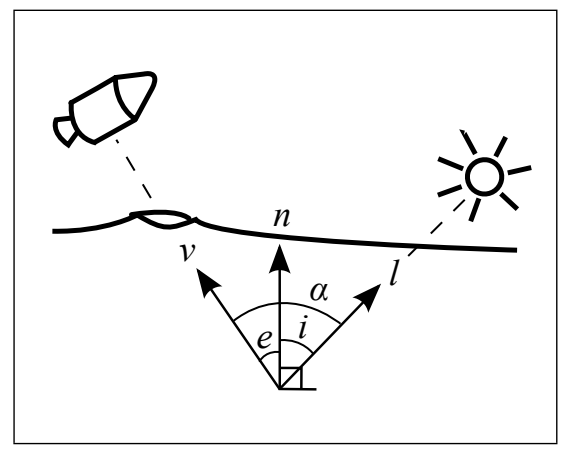

Fig. 2. Illumination and viewing angles used by the LunarLambertian reflectance model.

\section{PARAMETER ESTIMATION}

The computation of the reflectance ("R Map" in Figure 1) using Equation 1 is central to our albedo reconstruction technique. Let $I_{i j}, A_{i j}, R_{i j}$ be the Apollo Metric Camera image value, albedo and reflectance at pixel $i j$, and $T$ be a variable proportional to the exposure time of the image. Then

$$
I_{i j}=T A_{i j} R_{i j}
$$

Note that the image formation model described in Equation 2 does not take into consideration the camera transfer function since the influence of the non-linearities of the camera transfer function plays a secondary role in the image formation model [2]. From Equation 2 it can be seen that when the observed pixel value, exposure time, and reflectance value are known, the image formation model in Equation 2 provides a unique albedo value. However, these observed values are subject to errors arising from measurement (exposure time), image scanning process (image value) and stereo modeling errors (reflectance), resulting in imprecise albedo calculations. The method presented here mitigates these errors by reconstructing the albedo of the Lunar surface from all the overlapping images, along with their corresponding exposure times and DTM information. The albedo reconstruction is formulated as the least squares problem that minimizes the following cost function Q:

$$
\mathbf{Q}=\sum_{k} \sum_{i j}\left[\left(I_{i j}^{k}-A_{i j} T^{k} R_{i j}^{k}\right)^{2} S_{i j}^{k} w_{i j}^{k}\right]
$$

where the superscript $k$ denotes the variables associated with the $k$-th image and $S_{i j}^{k}$ is a shadow binary variable, $S_{i j}^{k}=1$ when the pixel is in shadow and 0 otherwise. The weights $w_{i j}^{k}$ are chosen such that for each image $k$ they have linearly decreasing values from the center of the image $\left(w_{i j}^{k}=1\right)$ to the image boundaries $\left(w_{i j}^{k}=0\right)$. The choice of these weights insures that the reconstructed albedo mosaic is seamless. The $S_{i j}^{k}$ values play an important role in accurate albedo estimation by discarding image pixels that are in shadow and for which the DTM and the reflectance models are unreliable $[1,4]$. The DTM used in computing the reflectance value $R_{i j}^{k}$ at each pixel is obtained by a weighted average (using the above weighting coefficients) of the DTM generated using stereo from all pairs of consecutive Apollo Metric Camera images. Figure 3 shows the DTM before and after the weighted DTM averaging and denoising process. It can be seen that the noise artifacts in the original DTM are reduced in the averaged DTM while the edges of the large craters and mountain regions are well-preserved. The color shaded averaged DTM of the Apollo 15,
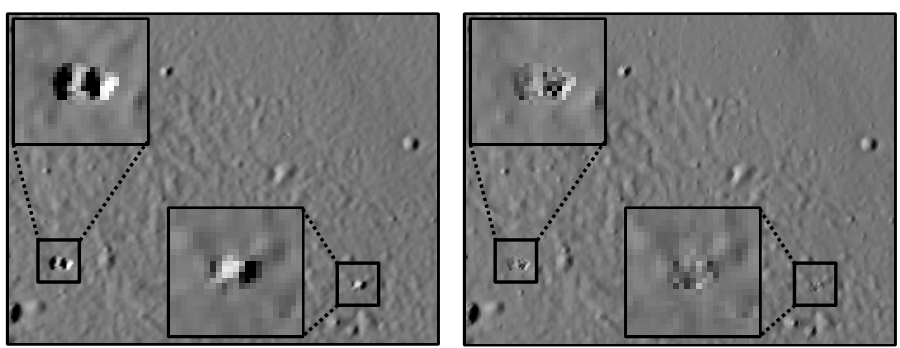

Fig. 3. Hill-shaded maps generated using (left) single local DTM and (right) denoised DTM derived from weighted average of overlapping DTMs. Our denoising approach preserves the structure while reducing the artifacts shown in the insets.

16 and 17 missions is shown in Figure 4 over the image mosaic of the Clementine mission.

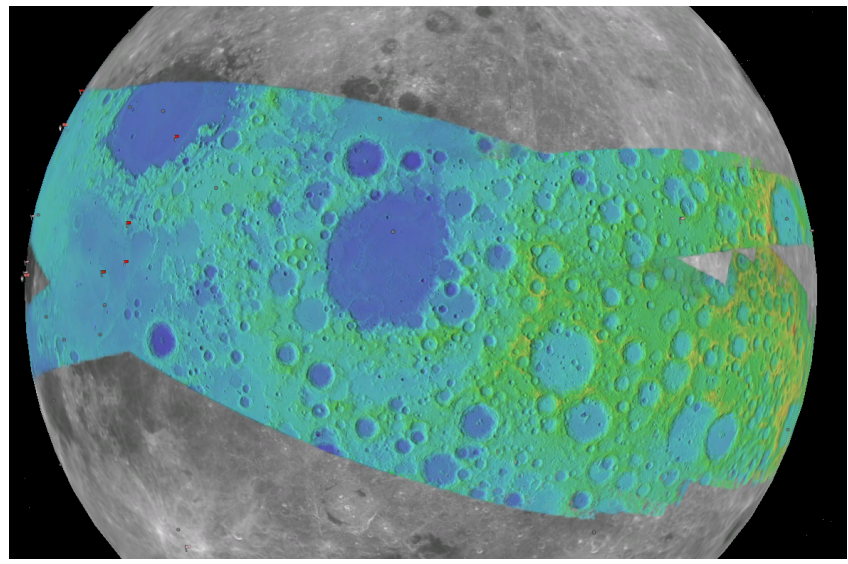

Fig. 4. Colorshade of the enhanced digital terrain model of the Apollo zone.

The optimal albedo reconstruction [10] from multiview images and their corresponding DTM is formulated as a minimization problem of finding

$$
\left\{\tilde{A}_{i j}, \tilde{T}^{k}, \tilde{c}_{l}\right\}=\arg \min _{A_{i j}, T^{k}, c_{l}} \mathbf{Q}
$$

for all pixels $i j$ and images $k$, where $\mathbf{Q}$ is the cost function in Equation 3. An iterative solution to the above least square problem is given by the Gauss-Newton updates given below.

- Step 1 (initialization): Compute the enhanced DTM as described earlier and the weights. Normalize the weights so that the sum of weights over all images is 1 at each pixel. Initialize the exposure time as inversely proportional to the average image reflectance. Initialize the phase coefficients $c_{1}$ and $c_{2}$ to some reasonable values. Initialize the albedo as the arg min of the cost function $\mathbf{Q}$ for fixed exposure time and phase coefficients

$$
A_{i j}=\frac{\sum_{k} I_{i j}^{k} T^{k} R_{i j}^{k} S_{i j}^{k} w_{i j}^{k}}{\sum_{k}\left(T^{k} R_{i j}^{k}\right)^{2} S_{i j}^{k} w_{i j}^{k}}
$$


- Step 2: Re-estimate the exposure time using

$$
\tilde{T}^{k}=T^{k}+\frac{\sum_{i j}\left(I_{i j}^{k}-A_{i j} T^{k} R_{i j}^{k}\right) A_{i j} R_{i j}^{k} S_{i j}^{k} w_{i j}^{k}}{\sum_{i j}\left(A_{i j} R_{i j}^{k}\right)^{2} S_{i j}^{k} w_{i j}^{k}}
$$

- Step 3: Re-estimate the phase coefficients using

$$
\tilde{c_{1}}=c_{1}+\frac{\sum_{i j k}\left(I_{i j}^{k}-A_{i j} T^{k} R_{i j}^{k}\right) A_{i j} T^{k} \frac{\partial R_{i j}^{k}}{\partial c_{1}} S_{i j}^{k} w_{i j}^{k}}{\sum_{i j k}\left(A_{i j} T^{k} \frac{\partial R_{i j}^{k}}{\partial c_{1}}\right)^{2} S_{i j}^{k} w_{i j}^{k}}
$$

and analogously for $c_{2}$. The partial derivatives $\partial R_{i j}^{k} / \partial c_{1}$ and $\partial R_{i j}^{k} / \partial c_{2}$ are computed from Equation 1.

- Step 4: Re-estimate the albedo using

$$
\tilde{A}_{i j}=A_{i j}+\frac{\sum_{k}\left(I_{i j}^{k}-A_{i j} T^{k} R_{i j}^{k}\right) T^{k} R_{i j}^{k} S_{i j}^{k} w_{i j}^{k}}{\sum_{k}\left(T^{k} R_{i j}^{k}\right)^{2} S_{i j}^{k} w_{i j}^{k}}
$$

- Step 5: Compute the error cost function $\mathbf{Q}$ for the re-estimated values of the albedo and exposure time.

- Convergence: If the error between consecutive iterations falls below a fixed threshold, then stop. Otherwise return to step 2.

- Step 6: Estimate the error map of the albedo reconstruction at each pixel using the formula

$$
E_{i j}=\frac{\sum_{k}\left(I_{i j}^{k} /\left(T^{k} R_{i j}^{k}\right)-A_{i j}\right)^{2} S_{i j}^{k} w_{i j}^{k}}{\sum_{k} w_{i j}^{k}}
$$

\section{EXPERIMENTAL RESULTS}

The main results of this research are the reconstruction of the Lunar albedo from images taken by the Apollo Metric Camera flown onboard the Apollo 15, 16 and 17 missions, and the corresponding error map. The reconstructed albedo (Figure 5) covers approximately $16 \%$ of the Lunar surface at an unprecedented resolution of 10 meters/pixel for this coverage. For comparison, LROWAC albedo mosaic covers $100 \%$ of the Lunar surface at 100 meters/pixel and the current LRO-NAC derived albedo mosaic covers about $2 \%$ of the Lunar surface at 1 meter/pixel resolution. Compared to imagery obtained from more recent missions, the images captured on film by the Apollo missions have more noise artifacts and errors in camera position/orientation and exposure time, making the processing of this data significantly more challenging.

The reconstructed albedo is shown in Figure 5 over the Clementine mission image mosaic. The images captured by the Clementine mission at 500 meters/pixel resolution were taken at very low incidence and emission angles (that is, both the Sun and the spacecraft were rather high in the sky above the terrain) making these images have an average intensity close to the average albedo. Note that the transition between the reconstructed albedo and the background Clementine data is practically seamless demonstrating that our approach can recover the average albedo intensity. However, the Clementine images do not describe a true albedo map since at each pixel the local emission and incidence angles are non-zero due to the irregular terrain shape.

Our approach significantly removes shadow and illumination artifacts in the input imagery. Note the shadow removal effect in Figure 7 (top-left), and the contour of crater showing the ejecta ring around its rim in the reconstructed albedo image (Figure 7 top-right). Figure 7 (bottom) shows the illumination removal effect in the reconstructed albedo for the Apollo 15 landing site. The illumination/shadow removal effect as well as the seam reduction is shown in Figure 8 (compare the left and right images). Pivotal to reducing the seam artifacts in the top-right image in Figure 8 was the use of the phase coefficients $c_{1}$ and $c_{2}$ in the reflectance formula, Equation 1.

The reconstruction error map (Figure 6) is computed at each pixel using Equation 9. Brighter values have higher estimated errors, which in turn implies a larger discrepancy between the pixel values at that location in the original overlapping images. It can be noticed that larger albedo reconstruction errors occur around crater ridges and are due to larger terrain reconstruction errors in these regions. Larger than average albedo reconstruction errors are also noticed at boundaries between orbits on a East-West Lunar traverse and are due to the small fraction of overlap between images taken in adjacent orbits compared to the overlap between consecutive images within the same orbit.

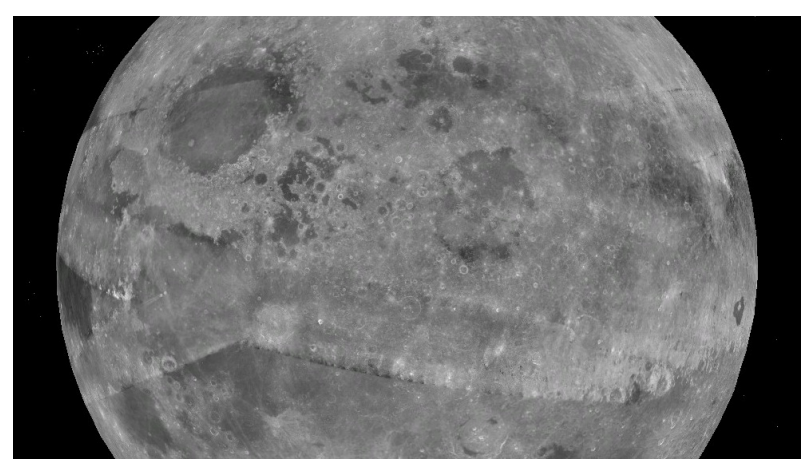

Fig. 5. Reconstructed albedo of the Apollo zone on top of Clementine imagery in Google Earth.

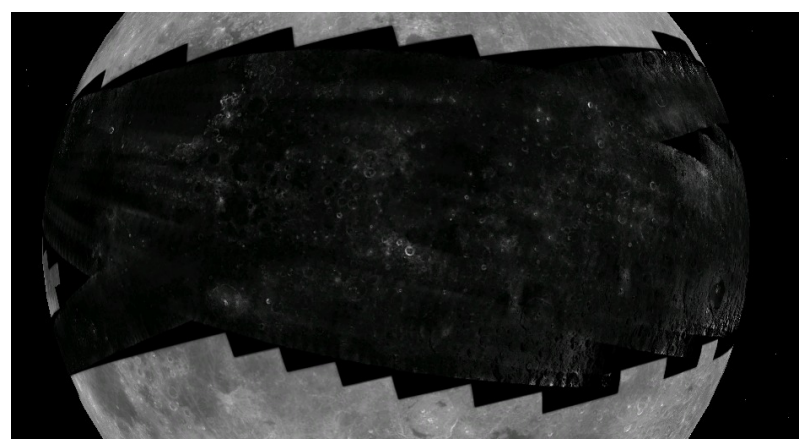

Fig. 6. Error map for the reconstructed albedo.

The Lunar albedo and the corresponding error map files can be accessed in GeoTiff tile format from

https://byss.arc.nasa.gov/albedo/albedo.html

and

https://byss.arc.nasa.gov/albedo/error.html 

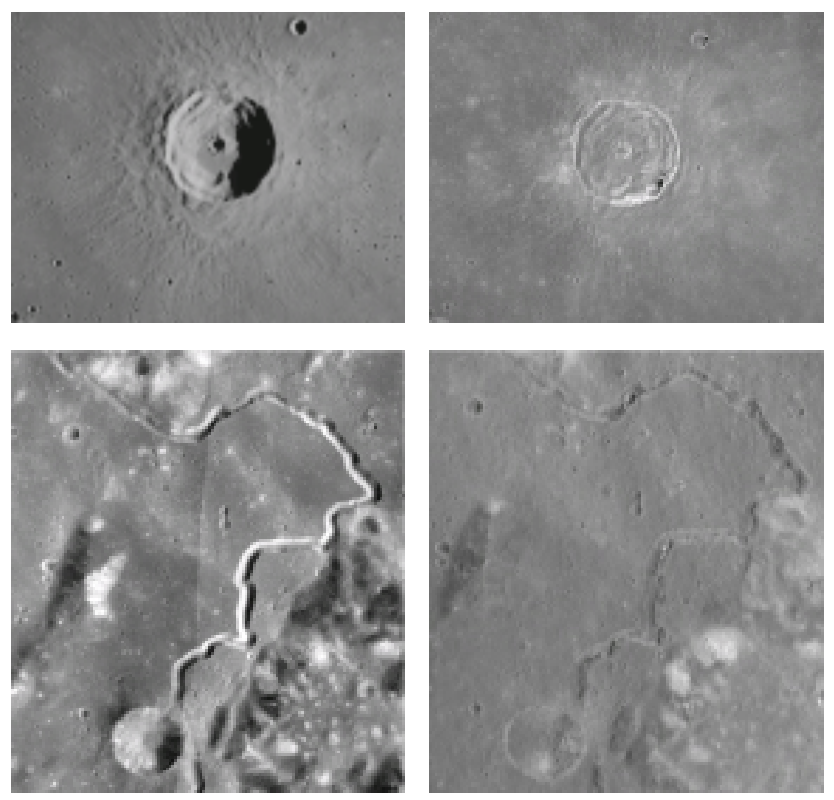

Fig. 7. Image mosaic of the Apollo zone (left), and the reconstructed albedo (right).
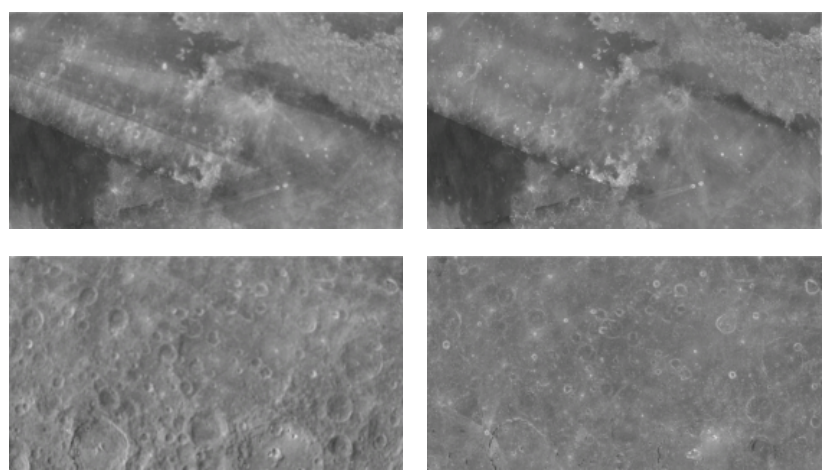

Fig. 8. Image mosaic of the Apollo zone (left), and the reconstructed albedo (right).

respectively. The albedo in KML format (viewable in Google Earth) can be accessed at

$$
\text { https://byss.arc.nasa.gov/albedo/albedo.kml }
$$

\section{CONCLUSIONS AND FUTURE WORK}

This work introduces a method for photometric reconstruction of the Lunar surface from archival images taken during the Apollo 15, 16 and 17 missions. The reconstructed area covers approximately $16 \%$ of the Lunar surface at an unprecedented resolution of 10 meters/pixel for this coverage. The proposed method jointly estimates the surface albedo and camera exposure time, and refines the Lunar photometric parameters using a weighted least squares method. The reconstructed albedo and reconstruction error are made publicly available, and it is hoped that they will help planetary scientists and mission planners in current and future NASA missions. The methods introduced in this paper also enable future research in robust surface reconstruction and shape-from-shading techniques that will further increase the resolution of current terrain models.

\section{REFERENCES}

[1] V. Arévalo, J. González, and G. Ambrosio. Shadow detection in colour high-resolution satellite images. Int. J. Remote Sens., 29(7):1945-1963, 2008.

[2] R. W. Gaskell, O. S. Barnouin-Jha, D. J. Scheeres, A. S. Konopliv, T. Mukai, S. Abe, J. Saito, M. Ishiguro, T. Kubota, T. Hashimoto, J. Kawaguchi, M. Yoshikawa, K. Shirakawa, T. Kominato, N. Hirata, and $\mathrm{H}$. Demura. Characterizing and navigating small bodies with imaging data. Meteoritics and Planetary Science, 43:1049-1061, September 2008.

[3] S. J. Lawrence, M. S. Robinson, M. Broxton, J. D. Stopar, W. Close, J. Grunsfeld, R. Ingram, L. Jefferson, S. Locke, R. Mitchell, T. Scarsella, M. White, M. A. Hager, T. R. Watters ad E. BowmanCisneros, J. Danton, and J. Garvin. The Apollo Digital Image Archive: New Research and Data Products. In Proc of the NLSI Lunar Science Conference, page 2066, 2008.

[4] Huertas. Matthies L. and Y. Cheng. Stereo vision and shadow analysis for landing hazard detection. IEEE International Conference on Robotics and Automation, pages 2735 - 2742, 2008.

[5] A. S. McEwen. Exogenic and endogenic albedo and color patterns on Europa. Journal of Geophysical Research, 91:8077-8097, July 1986.

[6] A. S. McEwen. Photometric functions for photoclinometry and other applications. Icarus, 92:298-311, August 1991.

[7] A. S. McEwen. A precise lunar photometric function. Lunar and Planet. Sci. Conf. 27th, 1996.

[8] M. Minnaert. The reciprocity principle in lunar photometry. Journal of Astrophysics, 1941.

[9] M.S. Robinson, E.M. Eliason, H. Hiesinger, B.L. Jolliff, A.S. McEwen, M.C. Malin, M.A. Ravine, D. Roberts, P.C. Thomas, and E.P. Turtle. LROC - Lunar Reconaissance Orbiter Camera. In Proc of the Lunar and Planetary Science Conference (LPSC) XXXVI, page 1576, March 2005.

[10] A. Yuille and D. Snow. Shape and albedo from multiple images using integrability. In CVPR '97: Proceedings of the 1997 Conference on Computer Vision and Pattern Recognition (CVPR '97), page 158, Washington, DC, USA, 1997. IEEE Computer Society. 